\title{
Aproximación socio-histórica y psicoanalítica del machismo y sexismo
}

\author{
Socio-historic and psychoanalytic approach to machism and sexism \\ Miriam Arteaga-Barba ${ }^{a}$, Karla G. López-Escamilla ${ }^{b}$, Kevin Monroy-Sánchez ${ }^{c}$, Josué R. Pérez- \\ León $^{d}$, Gelacio Guzmán-Díaz ${ }^{e}$, Jesús Cisneros-Herrera ${ }^{f}$
}

\begin{abstract}
:
In this paper, it is present a socio-historic and psychoanalytic approach to sexism and machism. The main idea is that social organization forms that helped human being to survive turned into commands for each sex, which values and meanings were attributed to. So, through time, changes in these social organizations were hindered by ideologies that justify the roles of men and women in the world and in society. As a result, sexism and machism became discourses that justify male domination over women. And these discourses, in turn, are introjected in psychic structure, so that change in individuals and societies is difficult to achieve. Machism builds up an ideal of man and woman, which everyone strives to be like in order to feel valued. Namely, machism plays and important role in the individual's narcisism.
\end{abstract}

Keywords:

Sexism, machism, micromachism, hostile micromachism, benevolent micromachism

\section{Resumen:}

En este trabajo se hace una revisión socio-histórica del sexismo y el machismo. La idea central es que las formas de organización social que permitieron la supervivencia del ser humano se convirtieron en obligaciones para cada sexo a las que se atribuyeron valoraciones y significados. De modo que, a través del tiempo, los cambios en la organización social se han visto obstruidos por la ideología que justifica el lugar de hombres y mujeres en el mundo y la sociedad. Así, sexismo y machismo se convirtieron en discursos que justifican el dominio del hombre sobre la mujer. Y estos discursos y esta dominación, a su vez, se introyectan en la estructura psíquica, por lo que el cambio en las personas y la sociedad resulta difícil. El machismo construye un ideal de hombre y de mujer al que se aspira parecerse para poder sentirse valorado, es decir, juega un papel importante en el narcisismo de los individuos.

Palabras Clave:

Sexismo, machismo, micromachismo, machismo hostil, machismo benevolente

\section{Introducción}

A lo largo de los últimos años, diversos análisis sociológicos han sustentado la idea de que la violencia machista afecta por igual a todas la mujeres independientemente de su clase, etnia u otras categorías de clasificación social. Además, se ha cuestionado la influencia y/o implicación que tienen el sexismo y el machismo en la actualidad, así como el papel de estos en la violencia de género, la cual es considerada uno de los principales problemas sociales (1). Por ello, este trabajo tiene como fin abordar el sexismo desde sus orígenes y expresiones, considerando que tiene un papel

\footnotetext{
a Autor de Correspondencia, Universidad Autónoma del Estado de Hidalgo, Escuela Superior de Atotonilco de Tula, https://orcid.org/0000-0002-1283-004, Email: ar358896@uaeh.edu.mx

${ }^{\mathrm{b}}$ Universidad Autónoma del Estado de Hidalgo, Escuela Superior de Atotonilco de Tula, https://orcid.org/0000-0002-5999-9117, Email: lo304920@uaeh.edu.mx

${ }^{\mathrm{c}}$ Universidad Autónoma del Estado de Hidalgo, Escuela Superior de Atotonilco de Tula, https://orcid.org/0000-0002-5055-2319, Email: mo356145@uaeh.edu.mx

d Universidad Autónoma del Estado de Hidalgo, Escuela Superior de Atotonilco de Tula, https://orcid.org/0000-0002-9371-3610, Email pe304770@uaeh.edu.mx

${ }^{\mathrm{e}}$ Universidad Autónoma del Estado de Hidalgo, Escuela Superior de Atotonilco de Tula, https://orcid.org/0000-0003-4869-8658, Email: gelacio_guzman@uaeh.edu.mx

${ }^{\mathrm{f}}$ Universidad Autónoma del Estado de Hidalgo, Escuela Superior de Atotonilco de Tula, https://orcid.org/0000-0003-1370-8086, Email: jesus_cisneros@uaeh.edu.mx
} 
fundamental para el entendimiento de la violencia de género. También se abordará de manera general al machismo y sus matices, como los son los micromachismos, los cuales pueden ser las expresiones más sutiles de violencia.

Uno de los principales fundamentos teóricos que se retoman es la teoría del sexismo ambivalente. Esta teoría aborda dos tipos de actitudes hacia las mujeres: hostil y/o benevolente, de las cuales se hablara más adelante. A su vez partiendo de ellas es como se estructura la violencia de género, término por el cual se retoma el tema de género, en el que se considera a la masculinidad y la feminidad como los principales campos a abordar tomando en cuenta que estos parten de los roles sociales que les son impuestos a cada uno (2).

El machismo puede definirse como una ideología derivada del sexismo que defiende y justifica la superioridad y el dominio del hombre sobre la mujer; en él se exaltan las cualidades masculinas, como agresividad, independencia y dominancia, mientras se estigmatizan las cualidades femeninas, como debilidad, dependencia y sumisión (3). El machismo ha provocado que la mujer sea discriminada en diferentes ámbitos injustificadamente y en casos extremos incluso se le haya negado los mismos derechos que a los varones. Tan solo basta con mirar a partir de qué año fue permitido que una mujer votara en México. Pero ¿de dónde viene el machismo? ¿Cómo se manifiesta? ¿Por qué es un problema? Esas son preguntas a las que se intentará dar respuesta a lo largo de este trabajo.

\section{Contexto socio-histórico del machismo y sexismo}

Antes de abordar el machismo, es necesario explicar el sexismo. El sexismo puede definirse como una actitud discriminatoria dirigida a las personas en virtud de su pertenencia a un determinado sexo biológico, en función del cual se asumen diferentes características y conductas (4). Es decir, generaliza ciertas características en función del sexo minimizando las características personales, de modo que justifica el trato adverso hacia mujeres $u$ hombres en algunos sectores de la sociedad.

En las sociedades antiguas, la forma en que las mujeres contribuían era principalmente mediante la reproducción y el de cuidado de los hijos, mientras que la producción de bienes y la confección de leyes correspondían en su mayoría a los varones. Por ello, la masculinidad paso a ser concebida como una orientación instrumental que implica realizar tareas y resolver problemas. Esto es un claro ejemplo de la masculinidad hegemónica, término que se refiere a la imagen de masculinidad de aquellos hombres que se encuentran en el poder (5), mientras que la feminidad fue concebida como una orientación de expresividad que implica una preocupación por conseguir el bienestar de los demás y la armonía (6).

Por lo anterior la idea de género hace referencia a la construcción social del hecho de ser hombre o mujer, las expectativas y valores, la interrelación entre hombres y mujeres y las diferentes relaciones de poder y subordinación existentes entre ellos en una sociedad determinada (7). En términos simples se definió el cómo, un varón o una mujer, deben comportarse para ser considerados como tales; es decir, ser hombre significaba fabricar, encargarse de las tareas que implicaban esfuerzo físico y de resolver los problemas que se presentaran, mientras que el ser mujer, se centraba principalmente en el mostrar emociones y cuidar de los demás.

En este sentido los roles de género también llevaron a la existencia de diferentes sistemas normativos para hombres y mujeres, Carol Gilligan fue la primera en proponer una diferencia entre las normas morales masculinas y femeninas según la cual los varones poseen una ética de justicia basada en el respeto a los derechos formales ajenos, mientras la ética de las mujeres se centra en el cuidado de otros. Según Gilligan la ética femenina se enfoca en "ser-para-otros" mientras que la masculina se centra en "ser-para-si" (8). En términos simples las mujeres deben enfocarse en el bienestar de otros, mientras que los hombres, si bien se les adjudica una responsabilidad hacia su familia, poseen mayor libertad al momento de perseguir aspiraciones personales en comparación con las mujeres.

Al ser los hombres los encargados de gobernar, se formó una sociedad patriarcal, es decir una organización donde lo masculino posee supremacía o dominio (9), esto llevo a la creación de una jerarquía entre sexos estimulando relaciones de dominio y opresión entre hombres y mujeres (8). De acuerdo con Errázuriz (8) un ejemplo de lo anterior se encuentra en la obra de Aristóteles, quien clasificó a la humanidad en dos tipos, los que «por naturaleza mandan» y «los que son mandados». Los primeros eran los varones libres y los segundos, las mujeres, los esclavos y los niños.

En la actualidad, las relaciones de género parten de una supuesta superioridad de lo masculino, la subordinación y devaluación de lo femenino e incluso la dominación de unos hombres sobre otros, todo atendiendo a un modelo hegemónico (10). Uno de los ejemplos en los que se ve reflejado esto es en lo mencionado por Benjamin (11), quien sugiere que el hombre rechaza lo femenino por temor a volverse similar a la mujer, puesto que ve a la mujer como un objeto a controlar; con base a lo anterior se retoma el hecho de que existe un modelo hegemónico o predominante de masculinidad (12). Como ejemplo de esto último podemos mencionar la creencia de los hombres no lloran al considerar que el llanto es una conducta propia de las mujeres, y la comparación de hombres con mujeres a manera de insulto. En una sociedad regida por una moral y normas basadas en la condición sexual, se minimiza a la mujer además de que ésta debe comportarse acorde a lo que se considera adecuado para el género femenino (13). En el caso de las sociedades occidentales, las normas se basan en la heterosexualidad, por lo que aquellos que no entren en ella son apartados y/o etiquetados.

Un estudio para la validación de la Escala de sexismo ambivalente y el estudio de sus propiedades psicométricas realizado en ciudad de México en 2005, encontró que existían fuertes relaciones entre el sexismo de la madre y el sexismo de sus hijos e hijas, aunque esta conexión es mucho más fuerte en relación con sus hijas mostrando como las mujeres también perpetúan las ideologías sexistas (14). 
La Escala de sexismo ambivalente mencionada previamente se basa en la teoría del mismo nombre propuesta por el profesor en ciencias sociales Peter Glick y la psicóloga social Susan Fiske en 1996. Según esta teoría en la ideología sexista coexisten dos actitudes de diferente tipo hacia las mujeres que permiten dividirlo en sexismo hostil y benevolente (14).

El sexismo hostil está compuesto por prejuicios y conductas discriminatorias basadas en la idea de que las mujeres son un grupo inferior (14). Se trata de una concepción de la mujer como un ser inmaduro e incapaz de subsistir por cuenta propia, mientras que los hombres son considerados como los únicos capaces de ocupar posiciones de poder. En el sexismo hostil, también se considera que las mujeres utilizan su atractivo sexual para dominar al sexo masculino, creencia asociada con la hostilidad hacia las mujeres por ser percibidas como seres seductores y manipuladores. De hecho, el refrán popular de "a las mujeres, ni todo el amor ni todo el dinero" se refiere a guardar reservas a la hora de establecer una relación amorosa con una mujer o confiarle dinero.

El sexismo benevolente por su parte concibe a las mujeres como seres limitados en algunos aspectos (15), lo cual lleva a estereotiparlas, pero a diferencia del sexismo hostil posee un tono afectivo positivo, por lo cual conduce a conductas que pueden categorizarse como prosociales (16). El sexismo benevolente se basa en la creencia de que las mujeres necesitan de la protección masculina debido a su incapacidad para subsistir por cuenta propia y por la misma razón el rol de proveedor debe recaer en el varón. También abarca la creencia de que las mujeres deben complementar a los hombres de ahí que entre las características atribuidas al rol femenino se encuentren la habilidad para el cuidado doméstico y la crianza de los hijos entre otras.

Además de considerar a las relaciones de pareja heterosexuales como las más íntimas en las que un hombre puede participar (15). El sexismo benevolente puede observarse en refranes como "a la mujer ni con el pétalo de una rosa" cuyo significado popular es que nunca se debe golpear a una mujer. Sin embargo si se analiza literalmente, da a entender que una mujer es un ser tan frágil que incluso el material más delicado, como lo es el pétalo de una rosa, podría llegar a lastimarla. Otra demostración común son las historias ya sea clásicas o modernas en las cuales la protagonista femenina debe ser salvada del peligro por el protagonista masculino.

\section{Las manifestaciones y consecuencias del machismo}

Ya habiendo explicado el origen del sexismo es momento de concentrarnos en el tema principal de este trabajo el machismo, el cual se centra en la siguiente cuestión ¿Cuál es la diferencia entre sexismo y machismo? El término sexismo abarcaría cualquier ideología basada en la asociación de características cognitivas, conductuales o de cualquier otro tipo con el sexo biológico, mientras que el machismo se refiere específicamente a una ideología según la cual el hombre es superior a la mujer. El machismo no solo establece distinciones entre las áreas masculinas y femeninas, sino que además las contrapone (17).

La combinación entre polarización y generalización lleva a la creación de los estereotipos de género según los cuales los individuos pertenecientes a un sexo especifico poseen facilidad para tareas concretas, pero carecen de las capacidades necesarias para realizar aquellas asociadas con el sexo opuesto. Por ejemplo, la creencia de que las mujeres son capaces de cuidar a un bebé, pero son incapaces de reparar un electrodoméstico, o de que un hombre es capaz de manejar un automóvil con mayor precisión que una mujer pero es incapaz de cocinar. Esta polarización de las actividades dificulta a hombres y mujeres el progresar en áreas típicamente asociadas con el sexo opuesto. La psicoanalista kleiniana Joan Rivière propone que cuando una mujer obtiene éxito en un campo típicamente asociado a los hombres, debe exacerbar su feminidad para usarla como si de una máscara se tratase, para así evitar una reprimenda por parte del sexo opuesto, que pueden interpretarlo como un intento de robarles su espacio (8).

La pregunta sería ¿cómo y en qué afecta el machismo? Se trata de una ideología con diferentes expresiones siendo las más radicales la agresión física, y también la más conocida, pero no es la única forma de expresión, también existen manifestaciones más sutiles, aunque no por ello menos peligrosas, son los llamados micromachismos también conocidos como pequeñas tiranías, terrorismo íntimo o violencia "blanda". Los micromachismos se tratan de prácticas de dominación y violencia masculina en la vida cotidiana, que se encuentran en los límites de la evidencia, y a su vez buscan imponer, mantener el dominio y una supuesta superioridad sobre la mujer, también son considerados un "caldo de cultivo" para las demás formas de violencia de género, pues pueden llevar a formas más peligrosas de maltrato como el psicológico, emocional, físico, sexual y económico (18).

Los micromachismos se desarrollan de forma sutil para pasar desapercibidos, no son denominados micro por ser poco importante, sino porque son cotidianos, están normalizados y aceptados socialmente y por ello son difíciles de reconocer (19), en un contexto de pareja estas conductas se manifiestan como formas de presión de baja intensidad (20). El cambio de violencia física a formas de agresión más sutiles puede atribuirse a los cambios que la sociedad ha sufrido en las últimas décadas, el machismo por lo menos en las clases medias y alta, ya no consiste en el desbordante culto a la masculinidad ni en la misoginia explícita que antes lo caracterizaban, ya no se trata de un rasgo personal sino de una forma de relación interpersonal, una serie de creencias y actitudes implícitas, ocultas bajo la superficie de la vida cotidiana (17).

Un estudio comparativo entre el machismo sexual y las conductas de autoeficiencia realizado en la ciudad de Monterrey encontró una correlación entre la generación de pertenencia y la ideología machista. Para su estudio los investigadores utilizaron una muestra de 565 participantes conformada por individuos de diferentes generaciones, la llamada generación silenciosa conformada por individuos con una edad de entre 87 y 68 años, la generación denominada como baby boomers 
cuyas edades oscilan entre los 66 y 50 años, la generación $x$ conformada por personas de 49 a 31 años y la generación milenio conformada por personas de 30 a 12 años. Los resultados de la escala de machismo sexual mostraron que la generación silenciosa obtuvo los puntajes más altos en la categoría machismo seguida por los baby boomers, la generación x y finalmente la generación milenio, aunque la diferencia entre las puntuaciones de las dos últimas es relativamente pequeña (21).

Este nuevo tipo de machismo se enfoca en la coerción y el control con conductas como establecer horas de llegada para exigir que la pareja traiga el celular consigo en todo momento o permitirle trabajar solo bajo ciertas condiciones. En este sentido la mujer ve disminuidas sus oportunidades de progresar en el ámbito laboral y en sus intereses personales, lo cual más allá de solo perjudicar a la víctima en cuestión afecta a la sociedad en general. Por ejemplo, una mujer con un título en ingeniería acude a una compañía de construcción para pedir empleo y compite por el mismo puesto con un hombre cuyos conocimientos y competencias están en un nivel inferior, es de esperar que la mujer recibirá el empleo pero contrario a eso él recibe el empleo por el simple hecho de ser varón, en este caso la mujer solo perdería un potencial empleo, ella podría seguir buscando con la posibilidad de encontrar empleo en otro lugar, sin embargo la empresa perdería una empleada competente y con ello las potenciales ganancias y beneficios que le habría traído a dicha empresa.

Que nuevas expresiones del machismo hayan surgido recientemente no significa que las previas hayan desaparecido, tal es el caso de la violencia de género. La razón por la cual ha sido empleada constantemente por los individuos machistas, como un mecanismo de control, es porque permite conseguir presencia e influencia sobre la mujer (22). Asimismo la perspectiva de género tiene un papel fundamental para el entendimiento de la violencia de género (1), un ejemplo de ello es dónde la violencia es utilizada como medio para conseguir lo que se desea y dominar al sexo "débil"; actualmente la violencia de género se encuentra presente en los medios de comunicación, las agencias políticas y otras instancias sociales (2).

\section{El machismo desde la perspectiva psicoanalítica}

Como se mencionó previamente el machismo en una ideología presente en varias generaciones y la teoría freudiana ofrece una explicación sobre el mecanismo por medio del cual el sistema patriarcal se ha perpetuado. Freud propone que la construcción de la moral es un proceso que ocurre en el superyó, y que proviene de los mandatos explícitos y de la instancia moral inconsciente de ambos padres, que a su vez proviene de sus propios padres. De ese modo se perpetúan las tradiciones a lo largo de las generaciones (8).

Juliet Mitchell asegura que la teoría de Freud es de un orden simbólico falocéntrico interiorizado, la cual impone en los miembros de una sociedad características masculinas y femeninas con base a las creencias y tradiciones que se remontan siglos atrás. Mitchell aclara que lo anterior puede entenderse con base en la teoría freudiana, aunque nunca fue mencionado explícitamente por el propio Freud (8).

Antes de continuar sería necesario aclarar que dada la extensión y complejidad de la psique humana no resulta extraño que dentro de la comunidad psicoanalítica existan diferentes posturas e hipótesis que buscan explicar lo mismo sin que sea posible determinar cuál posee mayor veracidad. Por lo cual en este trabajo se considera a las teorías mencionadas a continuación como diferentes perspectivas sin dar mayor crédito a alguna. Jessica Benjamin propone una teoría interesante pero antes de abordarla sería necesario explicar el proceso de individuación infantil. Desde la perspectiva clásica la relación entre la madre y el infante es considerada como una relación entre dos sujetos que a la vez son dos objetos de amor recíproco y el niño logra autonomizarse cuando logra interiorizar los objetos (personas) con los que se identifica. La perspectiva de Benjamin sostiene que «la bifurcación cultural profundamente arraigada de toda la experiencia en los polos del género organizado heterosexualmente debilita el reconocimiento de la subjetividad de la madre y perpetúa la fantasía de la omnipotencia materna», lo que contribuye al repudio que hace el infante en el momento del complejo de Edipo y su vuelta hacia el padre (8). En este caso el infante considera a su madre como un ser todo poderosos que en un inicio lo protege sin embargo al crecer la cultura en la cual es criado le enseña que la mujer es un ser potencialmente peligroso por lo cual en niño pasa de verla como una figura protectora a percibirla como una figura amenazante por lo cual pasa a temerle y odiarla prefiriendo ser semejante a su padre perpetuando el proceso a lo largo de las generaciones.

En la sociedad moderna cada sujeto se adhiere a un orden simbólico, que al mismo tiempo se diversifica por la clase, la etnia, la cultura, pero que presenta un común denominador que es una heterodesignación por parte de un discurso sexo-genérico. En dicho discurso emitido durante siglos las mujeres no han tenido voz, o, por usar palabras de Braidotti, no han tenido acceso a un lugar en la enunciación (8). Los individuos que crecen en una cultura donde se considera que las mujeres deben ser subyugadas por los hombres y por lo mismo no consideran necesario o adecuado que las mujeres se expresen. Las propias mujeres interiorizan estas creencias criando a sus hijos e hijas bajo las mismas como mostraron los resultados de la Escala de sexismo ambivalente mencionada previamente perpetuando la ideología machista sin darse cuenta de ello o considerándolo como algo normal. Diferentes estudios han mostrado que el orden simbólico patriarcal ha sido interiorizado por las mujeres, contribuyendo a una autocensura en aspectos como la sexualidad el ejercicio del poder y la competitividad (8).

Emilce Dio Bleichmar propone que la sexualización de la imagen femenina que le viene impuesta por mandato de género y que contribuye a la escisión, clivaje, disociación en lo inconsciente de una tenaz resistencia a aceptar tal identidad. Tanto si es aceptada como rechazada será a costa de sufrimiento (8).

En este caso la mujer seria sexualizada por la sociedad de pertenencia representando un conflicto para las 
mujeres pues aceptarlo implicaría contradecir sus creencias personales mientras que rechazar esta concepción implicaría un conflicto con el medio social. La propuesta teórica de Bleichmar podría aplicarse a otros aspectos de la vida cotidiana para ambos sexos pues atenerse a los preceptos sociales sin estar de acuerdo con ellos u oponerse a estos implicara un conflicto ya sea con los otros o con uno mismo.

Respecto a la construcción de las subjetividades a partir de sistema sexo-género el proceso puede dividirse en dos vectores uno horizontal que representa la internalización del sistema simbólico por parte del sujeto y un vector vertical que corresponde al modo y los avatares relacionales a través de los cuales se entra al sistema. Ambos vectores contribuyen a diseñar un esquema en que el proceso dinámico de transmisión consciente/inconsciente del orden simbólico por parte de los otros se va construyendo como una malla que se teje tanto con lo intrapsíquico como con lo intersubjetivo (8). Para el padre del psicoanálisis hombres y mujeres se encuentran en igualdad de condiciones al ingresar en el sistema sexo-género; sin embargo en el discurso que rodea al infante ya existe una diferencia y una simbología propia de la cultura en la cual se nace. La diferencia entre los sexos y atributos de género se manifiestan explícitamente en el discurso de instituciones como la familia, la escuela o las tradiciones. Por otro lado, podemos encontrar mensajes implícitos que se transmiten en las relaciones más íntimas entre padres e hijos/hijas, y en especial en aquellas proyecciones inconscientes que vehiculan genealógicamente los adultos más cercanos (no solo de la madre). Según John Money, la identidad de género ocurre alrededor de los dieciocho meses de vida mientras que para Dio Bleichmar el yo es, desde su origen, una representación del sí mismo/a genérico (8).

En su experiencia clínica, Errázuriz (8) observó en múltiples ocasiones una escisión del yo en sus pacientes femeninos en las cuales existía una separación entre el yo como persona y el yo como mujer cosa ausente en el caso de los varones. Errázuriz atribuye esto a la asimilación del género humano por el masculino pues el varón asimila al ser humano en toda la dimensión de su construcción subjetiva y la mujer pasa a ser considerada por el orden simbólico como la diferente, la otra. Se puede concluir que existe un terreno común en el que se encuentra el hombre en su totalidad y parcialmente la mujer como persona, siendo la mujer como mujer la que es excluida. En este caso hombres y mujeres nacen iguales pero la cultura en la cual nace va transformándolos en hombres y mujeres y al ser considerar el género humano un sinónimo del género masculino las niñas son apartadas al punto de verlas como un grupo ajeno. Finalmente, las mujeres internalizan esta diferenciación cuando las normas sociales y familiares se constituyen su superyó para transmitirlas a sus descendientes posteriormente.

\section{Conclusiones}

Tomando como referencia lo antes mencionado, se puede concluir que el yo de las mujeres se construiría de tal forma que se percibirían a sí mismas como individuos cuyo principal objetivo es el de cuidar a los otros. Esto basado en su valor y en la capacidad para poder mantener unida a la familia y cuidar de esta cuando llegara a ser necesario, mientras que su superyó estaría constituido con base en normas según las cuales tiene permitido expresar libremente sus sentimientos, pero verían reducidas sus metas a funciones de cuidado o aspiraciones laborales menores pues el deber de colocar el bienestar de otros como prioridad le dificultaría cumplir con sus metas y aspiraciones personales. Los hombres por su parte ven su yo construido de tal forma que se valorarían a sí mismos con base a aspectos como el éxito profesional, la capacidad para proveer a su familia y demostraciones de superioridad ante otros hombres y mujeres, con un superyó conformado por normas según las cuales debe presentar características como la agresividad, las cuales no solo están bien vistas, sino que forman parte de la masculinidad.

Este sistema trae desventajas para ambos sexos pues las mujeres por un lado ven limitadas sus oportunidades en ámbitos como el laboral o incluso el personal si este choca con las normas sociales llegando al punto de que algunas mujeres presentan una escisión del yo con su concepto de sí mismas por un lado y su concepto de mujer por el otro. Los hombres si bien no se ven afectados en la misma medida, sí sufren de algunos problemas entre ellos por ejemplo en la discriminación o burla de otros hombres cuando su conducta o aspiraciones no están acorde con la concepción popular o personal de masculinidad. En general los miembros de ambos sexos encuentran dificultades cuando se interesan por un campo o expresan características asociadas con el sexo opuesto limitando su potencial y contribuciones a la sociedad.

La forma de abordar esta problemática sería cambiando la forma en la cual hombres y mujeres perciben la masculinidad y feminidad es decir modificar no el sistema en si sino la relación que los individuos se relacionan con este.

\section{Referencias}

[1] De Alencar-Rodrigues R, Cantera L. Violencia de Género en la Pareja: Una Revisión Teórica. Psico. 2012; 43(1): 116-126.

[2] Justo C. Reseña: Apuntes sobre violencia de género. Política y Sociedad. 2011; 48(2): 403-405.

[3] Moral de la Rubia J, Ramos Basurto S. Machismo, victimización y perpetración en mujeres y hombres mexicanos. Estudios sobre las Culturas Contemporáneas. 2016; 22(43): 37-66.

[4] Garaigordobil, M., Aliri, J. (2011). Sexismo hostil y benevolente: relaciones con el autoconcepto, el racismo y la sensibilidad intercultural. Revista de Psicodidáctica, 16(2), 331-350. Recuperado de http://www.redalyc.org/articulo.oa?id=17518828008

[5] Kimmel, M. (1997). Homofobia, temor, vergüenza y silencio en la identidad masculina. Masculinidad/es. Poder y Crisis. Ediciones de las Mujeres, 24, 1-14.

[6] Zubieta E, Beramendi M, Sosa F, Torres A. Sexismo ambivalente, estereotipos y valores en el ámbito militar. Revista de Psicología. 2011; 29(1): 101-130. 
[7] Arellano R. Género, medio ambiente y desarrollo sustentable: un nuevo reto para los estudios de género. Revista de Estudios de Género La Ventana. 2003; 2(17): 79-106.

[8] Errázuriz P. Misoginia romántica, psicoanálisis y subjetividad femenina. Zaragoza, España: Prensa Universitaria de Zaragoza; 2012.

[9] Rottenbacher J. Sexismo ambivalente, paternalismo masculino e ideología política en adultos jóvenes de la ciudad de lima. Pensamiento Psicológico. 2010; 7(14): 9-18.

[10] Menjívar M. Masculinidad y Poder. Espiga. 2001; 4: 1-8.

[11] Benjamin J. Los lazos de amor. Psicoanálisis, feminismo y el problema de la dominación. Buenos Aires, Argentina: Paidós; 1996.

[12] Peralta A. Los hombres mueren en silencio. México: Aljibe; 2013.

[13] Bochar Pizarro JE. Género y psicoanálisis. Cuernavaca, Morelos, México: Universidad Autónoma del Estado de Morelos, CICSER; 2018.

[14] Días C, Rosas M, Gonzales M. Escala de Machismo Sexual (EMSSexismo-12): diseño y análisis de propiedades psicométricas. Summa Psicológica UST. 2010; 7(2): 5-44.

[15] Cruz CE, Zempoaltecatl V, Correa FE. Perfiles de sexismo en la ciudad de México: validación del cuestionario de medición del sexismo ambivalente. Enseñanza e Investigación en Psicología. 2005; 10(2): 381-395.

[16] Expósito F, Glick P, Moya M. Sexismo ambivalente medición y correlatos. Revista de Psicología Social. 1998; 13(2): 159-169.

[17] Castañeda M. El machismo invisible: un enfoque interpersonal. Este País. 2002; 133: 50-55.

[18] Bonino Méndez L. Micromachismos: la violencia invisible en la pareja. Actualidad Psicológica. 1998; 254: 1-16.

[19] Jímenez Moyano MJ. Micromachismos: La violencia oculta. (Trabajo de Fin de Grado). Universidad de Jaén, España; 2016.

[20] Mayor V. Micromachismo en parejas jóvenes. (Trabajo de Fin de Grado). Universidad de Sevilla, España; 2013.

[21] Díaz C, González M. Comparación Intergeneracional del machismo sexual y conductas de autoeficacia. Acta de investigación psicológica. 2012; 2(2): 638-649.

[22] Expósito F. Violencia de género: La asimetría social en las relaciones entre mujeres y hombres favorece la violencia de género. Mente y Cerebro. 2011; 48: 20-25. 